\title{
ANALYTIC INTEGRABILITY OF BIANCHI CLASS A COSMOLOGICAL MODELS WITH $k=1$
}

\author{
ANTONI FERRAGUT ${ }^{1}$, JAUME LLIBRE ${ }^{2}$ AND CHARA PANTAZI ${ }^{3}$
}

\begin{abstract}
We complete the study of the analytic integrability of the Class A of Bianchi cosmological models with $k=1$, characterizing the analytic first integrals of the Bianchi types $\mathrm{VI}_{0}$ and $\mathrm{VIII}_{0}$.
\end{abstract}

\section{Introduction AND STATEMENT OF the MAin RESUlt}

A cosmological model describes the universe and is defined by the space time geometry (determined by a metric); the presence of the matter and its physical behaviour (determined by the energy-momentum tensor); and the interaction between geometry and matter (described through the Einstein's equations).

Friedmann in 1922 introduced the study of homogeneous cosmologies. For spatially homogeneous cosmologies the Einstein field equations can be written as an autonomous system of first order differential equations, see [19] and references therein.

Bianchi models describe space-times which are foliated by homogeneous hypersurfaces of constant time. Homogeneity requires a three dimensional isometry group (and so a three dimensional Lie algebra). Bianchi $[2,3]$ was the first to solve the problem of classifying three dimensional Lie algebras which are non-isomorphic. The classification is determined by the dimension $n$ of the algebra. There are nine types of models according to $n$ :

(a) $n=0$ : type I;

(b) $n=1$ : types II, III;

(c) $n=2$ : types IV, V, VI, VII;

(d) $n=3$ : types VIII, IX.

The types I,V and IX contain as special cases the Friedmann-Robertson-Walker universes, see [16].

If we consider $X_{1}, X_{2}, X_{3}$ an apropiate basis of the three dimensional Lie algebra, then the classification depends on a scalar $a \in \mathbb{R}$ and a vector $\left(n_{1}, n_{2}, n_{3}\right)$, with

2000 Mathematics Subject Classification. 34A05, 34A34, 34C14.

Key words and phrases. polynomial first integral, analytic first integral, Bianchi cosmological models.

All the authors are partially supported by the MICIIN/FEDER grant MTM2008-03437. A.F. is additionally supported by grants Juan de la Cierva, 2009SGR-410 and MTM2009-14163-C0202. J.L. is additionally partially supported by an AGAUR grant number 2009 SGR410 and by ICREA Academia. C.P. is additionally partially supported by the MICIIN/FEDER grant number MTM2009-06973 and by the AGAUR grant number 2009SGR859. 
$n_{i} \in\{-1,0,1\}$, such that

$$
\left[X_{1}, X_{2}\right]=n_{3} X_{3}, \quad\left[X_{2}, X_{3}\right]=n_{1} X_{1}-a X_{2}, \quad\left[X_{3}, X_{1}\right]=n_{2} X_{2}+a X_{1},
$$

where [, ] is the Lie bracket. In particular for $a=0$ we obtain the models of Class A and for $a \neq 0$ we obtain the models of Class B. For more details see Bogoyavlensky [4]. The Bianchi models of Class A are detailed in Table 1.

\begin{tabular}{|c||c|c|c|c|c|c|}
\hline Type & $\mathrm{I}$ & $\mathrm{II}$ & $\mathrm{VI}_{0}$ & $\mathrm{VII}_{0}$ & $\mathrm{VIII}$ & $\mathrm{IX}$ \\
\hline \hline$a$ & 0 & 0 & 0 & 0 & 0 & 0 \\
\hline$n_{1}$ & 0 & 1 & 1 & 1 & 1 & 1 \\
\hline$n_{2}$ & 0 & 0 & -1 & 1 & 1 & 1 \\
\hline$n_{3}$ & 0 & 0 & 0 & 0 & -1 & 1 \\
\hline
\end{tabular}

TABle 1. The classification of Bianchi Class A cosmologies.

Bianchi models as dynamical systems were first studied in 1971 by Collins [5]. A systematic study is due to Bogoyavlensky and collaborators [4]. According to Bogoyavlensky's work, the dynamics of Class A types can be reduced into a six dimensional autonomous differential system defined on a compact manifold with a boundary. Since then we find in the literature a big amount of works studying the dynamical behaviour of these cosmologies.

It is worth to mention that the most contraversory model seems to be the Bianchi IX model because of its chaotic (in some sense) behaviour. Many authors studied the integrability of this model. We must mention that the notion of integrability can have different meanings. Cushman and Sniatycki [6] proved that Bianchi IX is a locally integrable Hamiltonian system. Latifi, Musette and Conte [7] proved that this model is not integrable in the sense that it does not satisfy the Painlevé property. Morales-Ruiz and Ramis [15], using techniques of differential Galois theory, showed that the Bianchi IX model (as a complex Hamiltonian system) is not completely integrable (in the Liouville sense) with rational first integrals. Llibre and Valls in [8], using techniques of formal series, proved that any analytic first integral of Bianchi IX is a function of its Hamiltonian. The same authors in [9] proved that Bianchi VIII does not admit an analytic first integral different from the Hamiltonian using the Darboux theory of integrability. Maciejewski, Strelcyn and Szydłowski in [12] applied Morales-Ramis approach (and also Kovacic algorithm for a reduced system) to prove the non-meromorphic integrability of Bianchi VIII.

In [14] Class A was studied reducing the dimension of the system. In [17] the authors studied the vacuum Bianchi models of Class A, and using the zero level set of the Hamiltonian they reduced the dimension of the dynamical system (preserving the polynomial structure). In [18] there is a study about the algebraic integrability of Class A Bianchi models in vacuum and with dust and radiative matter. 
The Bianchi Class A cosmological models that we consider here are defined by the Hamiltonian

$$
H=\frac{1}{\left(q_{1} q_{2} q_{3}\right)^{(1-k) / 2}} \mathcal{H}
$$

where

$$
\begin{aligned}
\mathcal{H} & =-\left(p_{1}^{2} q_{1}^{2}+p_{2}^{2} q_{2}^{2}+p_{3}^{2} q_{3}^{2}\right)+2\left(p_{1} p_{2} q_{1} q_{2}+p_{1} p_{3} q_{1} q_{3}+p_{2} p_{3} q_{2} q_{3}\right) \\
& +\frac{1}{2}\left(n_{1} n_{2} q_{1} q_{2}+n_{1} n_{3} q_{1} q_{3}+n_{2} n_{3} q_{2} q_{3}\right) \\
& -\frac{1}{4}\left(n_{1}^{2} q_{1}^{2}+n_{2}^{2} q_{2}^{2}+n_{3}^{2} q_{3}^{2}\right) .
\end{aligned}
$$

Here we consider the case $k=1$ also analyzed in the works of Maciejewski and Szydłowski $[13,14]$, and of Llibre and Valls $[8,9]$. See the first two papers for a good physical introduction to these systems.

The differential system associated to $\mathcal{H}$ is $\dot{q}_{i}=\mathcal{H}_{p_{i}}, \dot{p}_{i}=-\mathcal{H}_{q_{i}}$, for $i=1,2,3$, which writes

$$
\begin{aligned}
& \dot{q}_{1}=2 q_{1}\left(-p_{1} q_{1}+p_{2} q_{2}+p_{3} q_{3}\right) \\
& \dot{q}_{2}=2 q_{2}\left(p_{1} q_{1}-p_{2} q_{2}+p_{3} q_{3}\right) \\
& \dot{q}_{3}=2 q_{3}\left(p_{1} q_{1}+p_{2} q_{2}-p_{3} q_{3}\right) \\
& \dot{p}_{1}=-\frac{n_{1}}{2}\left(-n_{1} q_{1}+n_{2} q_{2}+n_{3} q_{3}\right)-2 p_{1}\left(-p_{1} q_{1}+p_{2} q_{2}+p_{3} q_{3}\right), \\
& \dot{p}_{2}=-\frac{n_{2}}{2}\left(n_{1} q_{1}-n_{2} q_{2}+n_{3} q_{3}\right)-2 p_{2}\left(p_{1} q_{1}-p_{2} q_{2}+p_{3} q_{3}\right), \\
& \dot{p}_{3}=-\frac{n_{3}}{2}\left(n_{1} q_{1}+n_{2} q_{2}-n_{3} q_{3}\right)-2 p_{3}\left(p_{1} q_{1}+p_{2} q_{2}-p_{3} q_{3}\right) .
\end{aligned}
$$

System (2) is Hamiltonian with three degrees of freedom. Due to its Hamiltonian structure it is completely integrable if it admits three independent first integrals in involution, see for more details [1].

Under the two changes of variables $q_{i} \rightarrow x_{i}, p_{i} \rightarrow x_{i+3} / x_{i}$, for $i=1,2,3$, first, and $x_{i} \rightarrow 6 x_{i}$, for $i=1,2,3, t \rightarrow t / 2$, afterwards, system (2) becomes

$$
\begin{aligned}
& \dot{x}_{1}=x_{1}\left(-x_{4}+x_{5}+x_{6}\right), \\
& \dot{x}_{2}=x_{2}\left(x_{4}-x_{5}+x_{6}\right), \\
& \dot{x}_{3}=x_{3}\left(x_{4}+x_{5}-x_{6}\right), \\
& \dot{x}_{4}=9 n_{1} x_{1}\left(n_{1} x_{1}-n_{2} x_{2}-n_{3} x_{3}\right), \\
& \dot{x}_{5}=9 n_{2} x_{2}\left(-n_{1} x_{1}+n_{2} x_{2}-n_{3} x_{3}\right), \\
& \dot{x}_{6}=9 n_{3} x_{3}\left(-n_{1} x_{1}-n_{2} x_{2}+n_{3} x_{3}\right) .
\end{aligned}
$$

This system has lost the Hamiltonian structure. The first integral corresponding to system (3) coming from (1) is

$$
\begin{aligned}
\mathcal{H}^{*} & =-9\left(n_{1}^{2} x_{1}^{2}+n_{2}^{2} x_{2}^{2}+n_{3}^{2} x_{3}^{2}\right)-\left(x_{4}^{2}-x_{5}^{2}-x_{6}^{2}\right) \\
& +18\left(n_{1} n_{2} x_{1} x_{2}+n_{1} n_{3} x_{1} x_{3}+n_{2} n_{3} x_{2} x_{3}\right)+2\left(x_{4} x_{5}+x_{4} x_{6}+x_{5} x_{6}\right) .
\end{aligned}
$$


Our aim in this work is to complete the characterization of the analytic integrability of all Bianchi cosmologies of Class A with $k=1$ described by systems (2) or (3).

Theorem 1. The following statements hold for system (3).

(a) The Bianchi type I model has three independent polynomial first integrals, namely $x_{4}, x_{5}$ and $x_{6}$.

(b) The Bianchi type II model has three independent polynomial first integrals, namely $x_{5}, x_{6}$ and $\mathcal{H}^{*}=-9 x_{1}^{2}-x_{4}\left(x_{4}-2 x_{5}-2 x_{6}\right)$.

(c) The Bianchi types $V I_{0}$ and VII $I_{0}$ models have the polynomial first integrals $x_{6}$ and $\mathcal{H}^{*}=-9\left(x_{1}-n_{2} x_{2}\right)^{2}-\left(x_{4}^{2}+x_{5}^{2}+x_{6}^{2}\right)+2\left(x_{4} x_{5}+x_{4} x_{6}+x_{5} x_{6}\right)$. These models do not admit any additional analytic first integral independent from the two previous ones.

(d) The Bianchi type VIII and IX models have the polynomial first integral $\mathcal{H}^{*}=$ $-9\left(x_{1}^{2}+x_{2}^{2}+n_{3}^{2} x_{3}^{2}\right)-\left(x_{4}^{2}+x_{5}^{2}+x_{6}^{2}\right)+18\left(x_{1} x_{2}+n_{3} x_{1} x_{3}+n_{3} x_{2} x_{3}\right)+2\left(x_{4} x_{5}+\right.$ $\left.x_{4} x_{6}+x_{5} x_{6}\right)$. These models do not admit any additional analytic first integral independent from the previous one.

Due to the Theorem of Liouville-Arnold (see for details [1]) and since system (2) is Hamiltonian, we only need three independent first integrals in involution for describing their dynamics. We provide the independent analytical first integrals of the equivalent system (3), from these we can compute the corresponding ones of system (2). Of course, systems (2) and (3) can have more than three independent first integrals.

Statements (a) and (b) of Theorem 1 are easy to check by direct computation. In fact the Bianchi type I model has the two additional non-polynomial independent first integrals

$$
x_{1}^{x_{4}-x_{5}+x_{6}} x_{2}^{x_{4}-x_{5}-x_{6}}, \quad x_{1}^{x_{4}+x_{5}-x_{6}} x_{3}^{x_{4}-x_{5}-x_{6}}
$$

and the Bianchi type II model has the two additional non-polynomial independent first integrals

$x_{1}^{\sqrt{A}-2 x_{6}} x_{2}^{\sqrt{A}}\left(A+\sqrt{A}\left(-x_{4}+x_{5}+x_{6}\right)\right)^{2 x_{6}}, \quad x_{1}^{\sqrt{A}-2 x_{5}} x_{3}^{\sqrt{A}}\left(A+\sqrt{A}\left(-x_{4}+x_{5}+x_{6}\right)\right)^{2 x_{5}}$,

where $A=\left(x_{5}+x_{6}\right)^{2}-\mathcal{H}^{*}$.

Statement (d) of Theorem 1 is proved in $[8,9]$.

The main result of this paper is statement (c) of Theorem 1 on the Bianchi types $\mathrm{VI}_{0}$ and $\mathrm{VII}_{0}$ models. Related with the integrability of these two systems there are some related results when the dynamics of these systems are reduced to dimension three, see $[14,10]$. 


\section{Proof of statement (c) of Theorem 1}

System (3) for Bianchi types $\mathrm{VI}_{0}$ and $\mathrm{VII}_{0}$ has the form

$$
\begin{aligned}
& \dot{x}_{1}=x_{1}\left(-x_{4}+x_{5}+x_{6}\right), \\
& \dot{x}_{2}=x_{2}\left(x_{4}-x_{5}+x_{6}\right), \\
& \dot{x}_{3}=x_{3}\left(x_{4}+x_{5}-x_{6}\right), \\
& \dot{x}_{4}=9 x_{1}\left(x_{1}-n_{2} x_{2}\right), \\
& \dot{x}_{5}=-9 n_{2} x_{2}\left(x_{1}-n_{2} x_{2}\right), \\
& \dot{x}_{6}=0,
\end{aligned}
$$

with $n_{2}= \pm 1$ depending on the Bianchi type.

The following result is well known, see for instance [11].

Proposition 2. Let $F$ be an analytic function and let $F=\sum_{i} F_{i}$ be its decomposition into homogeneous polynomials of degree $i$. Then $F$ is an analytic first integral of the homogeneous differential system (3) if and only if for all $i F_{i}$ is a homogeneous polynomial first integral of system (3).

Proposition 2 shows that for studying the analytic first integrals of the homogeneous differential system (3) it is sufficient to study its homogeneous polynomial first integrals.

We assume from now on that $n_{1}=1, n_{2}= \pm 1, n_{3}=0$; this case corresponds to Bianchi types $\mathrm{VI}_{0}$ and $\mathrm{VII}_{0}$, see Table 1 . Proposition 3 shows the existence of two independent polynomial first integrals and Theorem 4 assures that there are no more polynomial first integrals independent from the ones provided by Proposition 3.

Proposition 3. Two independent polynomial first integrals for the Bianchi types $V I_{0}$ and $V_{1} I_{0}$ models are $x_{6}$ and

$$
H=-9\left(x_{1}-n_{2} x_{2}\right)^{2}-\left(x_{4}^{2}+x_{5}^{2}+x_{6}^{2}\right)+2\left(x_{4} x_{5}+x_{4} x_{6}+x_{5} x_{6}\right) .
$$

Proof. It follows easily by direct computations.

Theorem 4. Let $h\left(x_{1}, \ldots, x_{6}\right)$ be a polynomial first integral of system (5). Then $h$ is a polynomial in the variables $x_{6}$ and $H$, where $H$ is given by (6).

We state a lemma before proving Theorem 4. See [9] for a proof.

Lemma 5. Let $x_{k}$ be one dimensional variables for $k=1, \ldots, n$ and $n>1$. Let $f=f\left(x_{1}, \ldots, x_{n}\right)$ be a polynomial and let $f_{1}=\left.f\left(x_{1}, \ldots, x_{n}\right)\right|_{x_{l}=c_{0}}$, for some $l \in$ $\{1, \ldots, n\}$, where $c_{0}$ is a constant. Then there exists a polynomial $g=g\left(x_{1}, \ldots, x_{n}\right)$ such that $f=f_{1}+\left(x_{l}-c_{0}\right) g$.

Proof of Theorem 4. Let $h_{1}=\left.h\right|_{x_{3}=0}$. We consider the involution $\sigma$ defined by $\sigma\left(x_{1}\right)=n_{2} x_{2}, \sigma\left(x_{2}\right)=n_{2} x_{1}, \sigma\left(x_{3}\right)=-x_{3}, \sigma\left(x_{4}\right)=x_{5}, \sigma\left(x_{5}\right)=x_{4}$ and $\sigma\left(x_{6}\right)=x_{6}$. We note that $h_{1}$ is invariant under the involution $\sigma$, namely $\sigma\left(h_{1}\right)=h_{1}$. By Lemma 
5 there exists a polynomial $g$ such that $h=h_{1}+x_{3} g$. System (5) on $x_{3}=0$ writes

$$
\begin{aligned}
& \dot{x}_{1}=x_{1}\left(-x_{4}+x_{5}+x_{6}\right), \\
& \dot{x}_{2}=x_{2}\left(x_{4}-x_{5}+x_{6}\right), \\
& \dot{x}_{4}=9 x_{1}\left(x_{1}-n_{2} x_{2}\right), \\
& \dot{x}_{5}=-9 n_{2} x_{2}\left(x_{1}-n_{2} x_{2}\right), \\
& \dot{x}_{6}=0 .
\end{aligned}
$$

Moreover $h_{1}$ is a first integral of system (7). Now we restrict system (7) to $x_{1}=0$ and we obtain

$$
\begin{aligned}
& \dot{x}_{2}=x_{2}\left(x_{4}-x_{5}+x_{6}\right), \\
& \dot{x}_{4}=0, \\
& \dot{x}_{5}=9 x_{2}^{2}, \\
& \dot{x}_{6}=0 .
\end{aligned}
$$

Let $h_{2}=\left.h_{1}\right|_{x_{1}=0} ; h_{2}$ is a polynomial first integral of system (8). Let $\tilde{H}=\left.H\right|_{x_{1}=0}=$ $-9 x_{2}^{2}-\left(x_{4}^{2}+x_{5}^{2}+x_{6}^{2}\right)+2\left(x_{4} x_{5}+x_{4} x_{6}+x_{5} x_{6}\right)$. It is clear that $\tilde{H}, x_{4}$ and $x_{6}$ are first integrals of system (8), and that they are independent. Since system (8) is a four dimensional system with three independent first integrals $\tilde{H}, x_{4}$ and $x_{6}$, we have $h_{2}=h_{2}\left(x_{4}, x_{6}, \tilde{H}\right)$. Applying Lemma 5 we get $h_{1}=h_{2}\left(x_{4}, x_{6}, \tilde{H}\right)+x_{1} g_{1}$, where $g_{1}=g_{1}\left(x_{1}, x_{2}, x_{4}, x_{5}, x_{6}\right)$ is a polynomial and $h_{2}$ eventually can be zero. Since $\tilde{H}=H+9 x_{1}\left(x_{1}-2 n_{2} x_{2}\right)$ we have

$$
h_{1}=h_{2}\left(x_{4}, x_{6}, \tilde{H}\right)+x_{1} g_{1}=h_{2}\left(x_{4}, x_{6}, H\right)+x_{1} g_{2},
$$

for some polynomial $g_{2}=g_{2}\left(x_{1}, x_{2}, x_{4}, x_{5}, x_{6}\right)$.

We claim that $h_{2}$ does not depend on $x_{4}$. We prove the claim: as $x_{6}$ and $H$ are also invariant by $\sigma$ we have

$$
h_{2}\left(x_{4}, x_{6}, H\right)+x_{1} g_{2}=h_{1}=\sigma\left(h_{1}\right)=h_{2}\left(x_{5}, x_{6}, H\right)+n_{2} x_{2} \sigma\left(g_{2}\right) .
$$

Since the most left hand side and the most right hand side of the previous expression are equal, it follows that $h_{2}\left(x_{4}, x_{6}, H\right)=h_{2}\left(x_{5}, x_{6}, H\right)$. Therefore $h_{2}=h_{2}\left(x_{6}, H\right)$. In short the claim is proved.

We have $h_{1}=h_{2}\left(x_{6}, H\right)+x_{1} g_{2}$. Next we prove that $g_{2} \equiv 0$ proceeding by contradiction. Suppose that $g_{2} \not \equiv 0$ and write $x_{1} g_{2}=x_{1}^{\alpha} g_{3}$, with $\alpha \in \mathbb{N}, g_{3} \neq 0$ and $x_{1} \nmid g_{3}$. Let $X$ be the vector field associated to system (7), i.e.

$$
\begin{aligned}
X & =x_{1}\left(-x_{4}+x_{5}+x_{6}\right) \frac{\partial}{\partial x_{1}}+x_{2}\left(x_{4}-x_{5}+x_{6}\right) \frac{\partial}{\partial x_{2}} \\
& +9 x_{1}\left(x_{1}-n_{2} x_{2}\right) \frac{\partial}{\partial x_{4}}-9 n_{2} x_{2}\left(x_{1}-n_{2} x_{2}\right) \frac{\partial}{\partial x_{5}} .
\end{aligned}
$$


As $h_{1}=h_{2}\left(x_{6}, H\right)+x_{1}^{\alpha} g_{3}, x_{6}$ and $H$ are first integrals of system (7) we have

$$
\begin{aligned}
0 & =\frac{X\left(h_{1}\right)}{x_{1}^{\alpha}}=\frac{X\left(x_{1}^{\alpha} g_{3}\right)}{x_{1}^{\alpha}} \\
& =x_{1}\left(-x_{4}+x_{5}+x_{6}\right) \frac{\partial g_{3}}{\partial x_{1}}+x_{2}\left(x_{4}-x_{5}+x_{6}\right) \frac{\partial g_{3}}{\partial x_{2}} \\
& +9 x_{1}\left(x_{1}-n_{2} x_{2}\right) \frac{\partial g_{3}}{\partial x_{4}}-9 n_{2} x_{2}\left(x_{1}-n_{2} x_{2}\right) \frac{\partial g_{3}}{\partial x_{5}}+\alpha\left(-x_{4}+x_{5}+x_{6}\right) g_{3} .
\end{aligned}
$$

Let $g_{4}=\left.g_{3}\right|_{x_{1}=0}$. Since $g_{4}$ satisfies equation (9) applied on $x_{1}=0$, we obtain

$$
x_{2}\left(x_{4}-x_{5}+x_{6}\right) \frac{\partial g_{4}}{\partial x_{2}}+9 x_{2}^{2} \frac{\partial g_{4}}{\partial x_{5}}+\alpha\left(-x_{4}+x_{5}+x_{6}\right) g_{4}=0 .
$$

Thus $x_{2} \mid g_{4}$ and $g_{4}=x_{2}^{\beta} g_{5}$, with $\beta \in \mathbb{N}, g_{5} \not \equiv 0$ and $x_{2} \nmid g_{5}$. Hence

$$
x_{2}\left(x_{4}-x_{5}+x_{6}\right) \frac{\partial g_{5}}{\partial x_{2}}+9 x_{2}^{2} \frac{\partial g_{5}}{\partial x_{5}}+\left[\alpha\left(-x_{4}+x_{5}+x_{6}\right)+\beta\left(x_{4}-x_{5}+x_{6}\right)\right] g_{5}=0,
$$

and therefore $x_{2} \mid g_{5}$, a contradiction. Hence $g_{2} \equiv 0$ and $h_{1}=h_{2}\left(x_{6}, H\right)$.

Back to $h$, we proved that $h=h_{2}\left(x_{6}, H\right)+x_{3} g$. It remains to prove that $g \equiv 0$. Assume that $g \not \equiv 0$. Let $x_{3} g=x_{3}^{\gamma} \bar{g}_{1}$, with $\gamma \in \mathbb{N}, \bar{g}_{1} \not \equiv 0$ and $x_{3} \nmid \bar{g}_{1}$. Let $Y$ be the vector field associated to system (5), i.e.

$$
\begin{aligned}
Y & =x_{1}\left(-x_{4}+x_{5}+x_{6}\right) \frac{\partial}{\partial x_{1}}+x_{2}\left(x_{4}-x_{5}+x_{6}\right) \frac{\partial}{\partial x_{2}} \\
& +x_{3}\left(x_{4}+x_{5}-x_{6}\right) \frac{\partial}{\partial x_{3}}+9 x_{1}\left(x_{1}-n_{2} x_{2}\right) \frac{\partial}{\partial x_{4}}-9 n_{2} x_{2}\left(x_{1}-n_{2} x_{2}\right) \frac{\partial}{\partial x_{5}} .
\end{aligned}
$$

As $h, x_{6}$ and $H$ are first integrals of system (5), we have

$$
\begin{aligned}
0 & =\frac{Y(h)}{x_{3}^{\gamma}}=\frac{Y\left(x_{3}^{\gamma} \bar{g}_{1}\right)}{x_{3}^{\gamma}} \\
& =x_{1}\left(-x_{4}+x_{5}+x_{6}\right) \frac{\partial \bar{g}_{1}}{\partial x_{1}}+x_{2}\left(x_{4}-x_{5}+x_{6}\right) \frac{\partial \bar{g}_{1}}{\partial x_{2}}+x_{3}\left(x_{4}+x_{5}-x_{6}\right) \frac{\partial \bar{g}_{1}}{\partial x_{3}} \\
& +9 x_{1}\left(x_{1}-n_{2} x_{2}\right) \frac{\partial \bar{g}_{1}}{\partial x_{4}}-9 n_{2} x_{2}\left(x_{1}-n_{2} x_{2}\right) \frac{\partial \bar{g}_{1}}{\partial x_{5}}+\gamma\left(x_{4}+x_{5}-x_{6}\right) \bar{g}_{1} .
\end{aligned}
$$

Let $\bar{g}_{2}=\left.\bar{g}_{1}\right|_{x_{3}=0}$. We have that $\bar{g}_{2} \not \equiv 0$ and that it satisfies equation (10) with $x_{3}=0$ :

$$
\begin{aligned}
& x_{1}\left(-x_{4}+x_{5}+x_{6}\right) \frac{\partial \bar{g}_{2}}{\partial x_{1}}+x_{2}\left(x_{4}-x_{5}+x_{6}\right) \frac{\partial \bar{g}_{2}}{\partial x_{2}}+9 x_{1}\left(x_{1}-n_{2} x_{2}\right) \frac{\partial \bar{g}_{2}}{\partial x_{4}} \\
& -9 n_{2} x_{2}\left(x_{1}-n_{2} x_{2}\right) \frac{\partial \bar{g}_{2}}{\partial x_{5}}+\gamma\left(x_{4}+x_{5}-x_{6}\right) \bar{g}_{2}=0 .
\end{aligned}
$$


We write $\bar{g}_{2}=x_{1}^{\delta} \bar{g}_{3}$, with $\delta \in \mathbb{N} \cup\{0\}, \bar{g}_{3} \not \equiv 0$ and $x_{1} \nmid \bar{g}_{3}$. Then, after dividing by $x_{1}^{\delta}$ we get

$$
\begin{aligned}
& x_{1}\left(-x_{4}+x_{5}+x_{6}\right) \frac{\partial \bar{g}_{3}}{\partial x_{1}}+x_{2}\left(x_{4}-x_{5}+x_{6}\right) \frac{\partial \bar{g}_{3}}{\partial x_{2}}+9 x_{1}\left(x_{1}-n_{2} x_{2}\right) \frac{\partial \bar{g}_{3}}{\partial x_{4}} \\
& -9 n_{2} x_{2}\left(x_{1}-n_{2} x_{2}\right) \frac{\partial \bar{g}_{3}}{\partial x_{5}}+\left[\gamma\left(x_{4}+x_{5}-x_{6}\right)+\delta\left(-x_{4}+x_{5}+x_{6}\right)\right] \bar{g}_{3}=0 .
\end{aligned}
$$

Let $\bar{g}_{4}=\left.\bar{g}_{3}\right|_{x_{1}=0}$. It satisfies equation (12) with $x_{1}=0$ :

$$
x_{2}\left(x_{4}-x_{5}+x_{6}\right) \frac{\partial \bar{g}_{4}}{\partial x_{2}}+9 x_{2}^{2} \frac{\partial \bar{g}_{4}}{\partial x_{5}}+\left[\gamma\left(x_{4}+x_{5}-x_{6}\right)+\delta\left(-x_{4}+x_{5}+x_{6}\right)\right] \bar{g}_{4}=0 .
$$

From this equation we have $x_{2} \mid \bar{g}_{4}$, and hence $\bar{g}_{4}=x_{2}^{\lambda} \bar{g}_{5}$, with $\lambda \in \mathbb{N}, \bar{g}_{5} \not \equiv 0$ and $x_{2} \nmid \bar{g}_{5}$. After dividing by $x_{2}^{\lambda}$ we obtain

$$
\begin{aligned}
& x_{2}\left(x_{4}-x_{5}+x_{6}\right) \frac{\partial \bar{g}_{5}}{\partial x_{2}}+9 x_{2}^{2} \frac{\partial \bar{g}_{5}}{\partial x_{5}} \\
& +\left[\gamma\left(x_{4}+x_{5}-x_{6}\right)+\delta\left(-x_{4}+x_{5}+x_{6}\right)+\lambda\left(x_{4}-x_{5}+x_{6}\right)\right] \bar{g}_{5}=0 .
\end{aligned}
$$

We note that the factor of $\bar{g}_{5}$ in (14) is

$$
(-\delta+\lambda+\gamma) x_{4}+(\delta-\lambda+\gamma) x_{5}+(\delta+\lambda-\gamma) x_{6} .
$$

The coefficients of $x_{4}, x_{5}, x_{6}$ in this expression are identically zero if and only if $\delta=$ $\lambda=\gamma=0$, which is not possible because $\gamma$ and $\lambda$ are different from zero. Hence we get from (14) that $x_{2} \mid \bar{g}_{5}$, a contradiction. Back to $h$, we have $g \equiv 0$ and then $h=h_{2}\left(x_{6}, H\right)$. The theorem follows.

Undoing the changes of variables, system (2) for Bianchi types $\mathrm{VI}_{0}$ and $\mathrm{VII}_{0}$ has only two independent polynomial first integrals which are $H_{1}=p_{3} q_{3}$ and $H_{2}=$ $-\left(q_{1}-n_{2} q_{2}\right)^{2}-4\left(p_{1}^{2} q_{1}^{2}+p_{2}^{2} q_{2}^{2}+p_{3}^{2} q_{3}^{2}\right)+8\left(p_{1} p_{2} q_{1} q_{2}+p_{1} p_{3} q_{1} q_{3}+p_{2} p_{3} q_{2} q_{3}\right)$, with $n_{2}= \pm 1$ depending on the Bianchi type. We note that we can obtain the Hamiltonian (1) from $\mathrm{H}_{2}: \mathcal{H}=\mathrm{H}_{2} / 4$.

Proposition 3 and Theorem 4 prove statement (c) of Theorem 1.

\section{REFERENCES}

[1] R. Abraham and J.E. Marsden, Foundations of mechanics. Second Edition, The BenjaminCummings Publishing Company, 1978.

[2] L. Bianchi, Sugli spazi a tre dimensioni che ammettono un gruppo continuo di movimenti (On the spaces of three dimensions that admit a continuous group of movements), Soc. Ital. Sci. Mem. di Mat. 11 (1898), 267-352.

[3] L. BiAnchi, Lezioni sulla teoria dei gruppi continui finiti di trasformazioni (Lectures on the theory of finite continuous transformation grups), Spoerri, Pisa (1918), 550-557.

[4] O.I. Bogoyavlensky, Qualitative Theory of Dynamical systems in Astrophysics and Gas Dynamics, Springer-Verlag, 1985.

[5] C.B. Collins, More Qualitative Cosmology, Comm. Math. Phys. 23 (1971), 137-158.

[6] R. Cushman and J. Sniatycki, Local integrability of the Mixmaster model, Reports on Math. Phys. 36 (1995), 75-89.

[7] A. Latifi, M. Musette And R. Conte, The Bianchi IX cosmological model is not integrable, Phys. Lect. A 194 (1994), 83-92. 
[8] J. Llibre And C. VAlls, Formal and analytical integrability of Bianchi IX system, J. Mat. Phys. 47 (2006), 022704-15 pp.

[9] J. Llibre AND C. VAlls, The Bianchi VIII model is neither global analytic nor Darboux integrable, J. Mat. Phys. 51 (2010), 092702-13 pp.

[10] J. Llibre and C. VAlLs, Nonintegrability of a class of the Bianchi $V I_{0}$ and VII $I_{0}$ models, J. of Geo. and Phys. 60 (2010), 815-822.

[11] J. Llibre And X. Zhang, Polynomial first integrals for quasi-homogeneous polynomial differential systems, Nonlinearity 15 (2002), 1269-1280.

[12] A.J. Maciejewski, J. Strelcyn and M. Szydeowski, Nonintegrability of Bianchi VIII Hamiltonian systems, J. Math. Phys. 42 (2001), 1728-13 pp.

[13] A.J. Maciejewski and M. SzydŁowski, On the integrability of Bianchi cosmological models, J. Phys. A: Math. Gen. 31 (1998), 2031-2043.

[14] A.J. Maciejewski And M. SzydŁowski, Bianchi Cosmologies as Dynamical systems, Celestial Mech. and Dyn. Astr. 73 (1999), 17-24.

[15] J.J. Morales-Ruiz and J.P. Ramis, Galoisian obstructions to integrability of Hamiltonian systems II, Meth. and Appl. of Anal. 8 (2001) 97-112.

[16] M. Ryan, Hamiltonian Cosmology, Lecture Notes in Physics 13, Springer Verlag, 1972.

[17] M. Szydeowski And J. Demaret, Bianchi A Cosmological Models as the Simplest Dynamical System in $\mathbb{R}^{4}$, Gen. Rel. and Grav. 31 (1999), 897-911.

[18] M. SzydŁOWski And M. Biesiada, Kovalevski Exponents and Integrability properties in Class A Homogeneous Cosmological Models, J. Nonlinear Math. Phys. 9 (2002), 1-10.

[19] J. Wainwright and G.F.R. Ellis, Dynamical systems in Cosmology, Cambridge University Press, 1997.

${ }^{1}$ Departament de Matemàtica Aplicada I, Universitat Politècnica de Catalunya (EtSeiB), Av. Diagonal, 647, 08028 Barcelona, Catalonia, Spain

E-mail address: antoni.ferragut@upc.edu

2 Departament de Matemàtiques, Universitat Autònoma de Barcelona (Edifici C), 08193 Bellaterra, Barcelona, Catalonia, Spain

E-mail address: jllibre@mat.uab.cat

3 Departament de Matemàtica Aplicada I, Universitat Politècnica de Catalunya (EPSEB), Av. Doctor Marañón, 44-50, 08028 Barcelona, Catalonia, Spain

E-mail address: chara.pantazi@upc.edu 